\title{
DOUBLY CHARMED MESONS
}

\author{
A. VALCARCE \\ Departamento de Fúsica Fundamental, Universidad de Salamanca \\ Plza. Merced $S / N$, Salamanca, E-37008, Spain \\ valcarce@usal.es \\ J. VIJANDE \\ Departamento de Física Atómica, Molecular y Nuclear, \\ Universidad de Valencia (UV) and IFIC (UV-CSIC) \\ Valencia, E-46100, Spain \\ javier.vijande@uv.es \\ T.F. CARAMÉS \\ Departamento de Fúsica Fundamental, Universidad de Salamanca \\ Plza. Merced S/N, Salamanca, E-37008, Spain \\ carames@usal.es
}

\begin{abstract}
Doubly charmed mesons are studied within a quark model framework. We solve the four-quark Schordinger equation by means of a variational approach by using different interacting potentials. Our results point to the existence of a stable isoscalar doubly charmed four-quark meson with quantum numbers $J^{P}=1^{+}$.
\end{abstract}

Keywords: mesons; exotics; tetraquarks.

PACS numbers: 14.40.Rt,21.30.Fe,12.39.Jh

The potentiality of the quark model for hadron physics in the low-energy regime became first manifest when it was used to classify the known hadron states. Describing hadrons as $q \bar{q}$ or $q q q$ configurations, their quantum numbers were correctly explained. This assignment was based on the comment by Gell-Mann ${ }^{1}$ introducing the notion of quark: 'It is assuming that the lowest baryon configuration (qqq) gives just the representations 1, 8 and 10, that have been observed, while the lowest meson configuration $(q \bar{q})$ similarly gives just 1 and 8 '. Since then, it has been assumed that these are the only two configurations involved in the description of physical hadrons. However, color confinement is also compatible with other multiquark structures, like the tetraquark $q q \bar{q} \bar{q}$ first introduced by $\mathrm{Jaffe}^{2}$.

The idea of unconventional quark structures is quite old and despite decades of progress, no exotic meson has been conclusively identified. A conclusive evidence of its existence seems rather difficult to be obtained with states that could be described by means of the lowest order configurations suggested by Gell-Mann, like the scalar 
mesons or uncharged charmonium resonances. In such cases, the experimental data will hardly exclude a theoretical model ${ }^{3}$. The study of exotic structures that cannot be accommodated in the scheme proposed by Gell-Mann should be of primary experimental and theoretical interest, because their existence would make manifest the contribution of four-quark states to hadron spectroscopy ${ }^{4}$. Among these exotic structures there are two types that could be experimentally accessible in present facilities: charged charmonium states ${ }^{5}$ and doubly heavy mesons.

Doubly heavy mesons is one of the first scenarios where the existence of bound multiquarks was proposed, systems composed of two light quarks and two heavy antiquarks $(n n \bar{Q} \bar{Q})$. Although they may be experimentally difficult to produce and also to detect ${ }^{6}$ it has been argued that for sufficiently large heavy quark mass they should be bound ${ }^{7}$. Their stability relies on the heavy quark mass. The heavier the quark the more effective the short-range Coulomb attraction to generate binding, in such a way that it could play a decisive role to bind the system. Moreover the $\bar{Q} \bar{Q}$ pair brings a small kinetic energy into the system contributing to stabilize it.

In this talk we revise the possible existence of stable doubly heavy mesons within the nonrelativistic quark model. For this purpose we solve the four-body Schrödinger equation based on a variational method in terms of generalized gaussians ${ }^{8}$. The fourquark wave function is written as a sum of outer products of color, isospin, spin and configuration terms

$$
\left|\phi_{C I S R}>=\right| \text { Color }>\mid \text { Isospin }>[\mid \text { Spin }>\otimes \mid R>]^{J M},
$$

such that the four-quark state is a color singlet with well defined parity, isospin and total angular momentum. Coupling the color states of two quarks (antiquarks) can yield two possible representations, the symmetric 6 -dimensional, $6(\overline{6})$, and the antisymmetric 3 -dimensional, $\overline{3}$ (3). Coupling the color states of the quark pair with that of the antiquark pair must yield a color singlet. Thus, there are only two possible color states for a $Q Q \bar{q} \bar{q}$ system,

$$
\text { |Color }>=\left\{\left|\overline{3}_{12} 3_{34}>,\right| 6_{12} \overline{6}_{34}>\right\} \text {. }
$$

These states have well defined symmetry under permutations. The spin states with such symmetry can be obtained in the following way,

$$
\mid \text { Spin }>=\left|\left(\left(s_{1}, s_{2}\right) S_{12},\left(s_{3}, s_{4}\right) S_{34}\right) S>=\right|\left(S_{12} S_{34}\right) S>.
$$

The same holds for the isospin, |Isospin $>=\mid\left(i_{3}, i_{4}\right) I_{34}>$, which applies only to the $n$-quarks, thus $I=I_{34}$.

To describe the spatial part of the wave function we choose for convenience the Jacobi coordinates,

$$
\begin{aligned}
& \overrightarrow{x_{1}}=\overrightarrow{r_{1}}-\overrightarrow{r_{2}}, \\
& \overrightarrow{x_{2}}=\vec{r}_{3}-\overrightarrow{r_{4}}, \\
& \overrightarrow{x_{3}}=\frac{m_{1} \vec{r}_{1}+m_{2} \vec{r}_{2}}{m_{1}+m_{2}}-\frac{m_{3} \vec{r}_{3}+m_{4} \vec{r}_{4}}{m_{3}+m_{4}},
\end{aligned}
$$


Using these vectors, it is easy to obtain basis functions that have well defined symmetry under permutations of the pairs (12) and (34). The radial wave function is expanded as a linear combination of generalized gaussians

$$
\mid R>=\sum_{i=1}^{n} \beta^{(i)} R^{i}\left(\vec{x}_{1}, \vec{x}_{2}, \vec{x}_{3}\right)=\sum_{i=1}^{n} \beta^{(i)} R^{i}
$$

where $n$ is the number of gaussians used for each color-spin-flavor component and $R^{i}$ depends on six variational parameters, $a^{i}, b^{i}, c^{i}, d^{i}, e^{i}$, and $f^{i}$, one for each scalar quantity: $\vec{x}_{1}^{2}, \vec{x}_{2}^{2}, \vec{x}_{3}^{2}, \vec{x}_{1} \cdot \vec{x}_{2}, \vec{x}_{1} \cdot \vec{x}_{3}$ and $\vec{x}_{2} \cdot \vec{x}_{3}$. Once the spin, color and flavor parts are integrated out the coefficients of the radial wave function are obtained by solving the system of linear equations

$$
\sum_{i} \beta^{(i)}\left[\left\langle R^{(j)}|H| R^{(i)}\right\rangle-E\left\langle R^{(j)} \mid R^{(i)}\right\rangle\right]=0 \quad \forall j,
$$

where the eigenvalues are obtained by a minimization procedure.

Assuming nonrelativistic quantum mechanics, the hamiltonian will be given by

$$
H=\sum_{i=1}^{4}\left(m_{i}+\frac{\vec{p}_{i}^{2}}{2 m_{i}}\right)+\sum_{i<j=1}^{4} V\left(\vec{r}_{i j}\right),
$$

where the potential $V\left(\vec{r}_{i j}\right)$ corresponds to an arbitrary two-body interaction. We have analyzed the possible existence of four-quark bound states by using two standard quark-quark interactions, a Bhaduri-like potential $(\mathrm{BCN})^{9}$ and a constituent quark model considering boson exchanges $(\mathrm{CQC})^{10}$. Both interactions give a reasonable description of the meson and the baryon spectroscopy, a thoughtful requirement considering that in the tetraquarks $q q$ and $q \bar{q}$ interactions will contribute.

We have performed an exhaustive analysis of the $Q Q \bar{q} \bar{q}$ spectra by means of the quark models described above. We have considered all isoscalar and isovector states with total orbital angular momentum $L \leq 1^{11}$. In Table 1 we show the results for some selected quantum numbers obtained with the CQC model. Our study draws a first general conclusion: once the four-body problem is properly solved the number of bound states is rather small if not zero. For the $c c \bar{n} \bar{n}$ only the $J^{P}=1^{+}(I=0)$ is bound independently of the interacting potential, already proposed more than twenty years ago in Ref. 7 .

We have also studied all ground states of the bottom sector using the same interacting potentials. It was pointed out in the early 80's that a $Q Q \bar{Q}^{\prime} \bar{Q}^{\prime}$ four-quark state should be stable against dissociation into $Q \bar{Q}^{\prime}+Q \bar{Q}^{\prime}$ if the ratio $m_{Q} / m_{Q^{\prime}}$ is large enough ${ }^{12}$. We observed that all bound states become deeper than in the charm sector and a new state, $J^{P}=0^{+}(I=0)$ appears, strengthening the conclusion that the larger the ratio of the quark masses the larger the binding energy.

In connection with the interacting potential used, it has been recently analyzed the stability of $Q Q \bar{n} \bar{n}$ and $Q \bar{Q} n \bar{n}$ systems in a simple string model ${ }^{13}$ considering only a multiquark confining interaction given by the minimum of a flip-flop or a butterfly potential in an attempt to discern whether confining interactions not factorizable as 
two-body potentials would influence the stability of four-quark states. The ground state of systems made of two quarks and two antiquarks of equal masses was found to be below the dissociation threshold. While for the cryptoexotic $Q \bar{Q} n \bar{n}$ the binding decreases when increasing the mass ratio $m_{Q} / m_{n}$, for the flavor exotic $Q Q \bar{n} \bar{n}$ the effect of mass symmetry breaking is opposite.

Table 1. Four-quark state properties for selected quantum numbers. All states have positive parity and total orbital angular momentum $L=0$. Energies are given in $\mathrm{MeV}$ and distances in fm. See text for details.

\begin{tabular}{c|ccccc}
\hline$\left(S_{T}, I\right)$ & $(0,1)$ & $(1,1)$ & $(1,0)$ & $(1,0)$ & $(0,0)$ \\
Flavor & $c c \bar{n} \bar{n}$ & $c c \bar{n} \bar{n}$ & $c c \bar{n} \bar{n}$ & $b b \bar{n} \bar{n}$ & $b b \bar{n} \bar{n}$ \\
\hline Energy $\left(E_{4 q}\right)$ & 3877 & 3952 & 3861 & 10395 & 10948 \\
Threshold $\left(T=M_{1}+M_{2}\right)$ & $D D \mid S$ & $D D^{*} \mid S$ & $D D^{*} \mid S$ & $\left.B B^{*}\right|_{S}$ & $\left.B_{1} B\right|_{P}$ \\
$\Delta_{E}=E_{4 q}-T$ & +5 & +15 & -76 & -217 & -153 \\
\hline$P_{M M}$ & 1.000 & - & - & - & 0.254 \\
$P_{M M^{*}}$ & - & 1.000 & 0.505 & 0.531 & - \\
$P_{M * M^{*}}$ & 0.000 & 0.000 & 0.495 & 0.469 & 0.746 \\
\hline$\left\langle x_{1}^{2}\right\rangle^{1 / 2}$ & 60.988 & 13.804 & 0.787 & 0.684 & 0.740 \\
$\left\langle x_{2}^{2}\right\rangle^{1 / 2}$ & 60.988 & 13.687 & 0.590 & 0.336 & 0.542 \\
$\left\langle x_{3}^{2}\right\rangle^{1 / 2}$ & 0.433 & 0.617 & 0.515 & 0.503 & 0.763 \\
$R S_{4 q}$ & 30.492 & 6.856 & 0.363 & 0.217 & 0.330 \\
\hline & & & &
\end{tabular}

Let us finally discuss the role played by hidden-color configurations, color singlets built by nonsinglet constituents. Besides Eq. (2), there are other two different ways for coupling two quarks and two antiquarks in a colorless state,

$$
\begin{aligned}
& {\left[\left(q_{1} \bar{q}_{3}\right)\left(q_{2} \bar{q}_{4}\right)\right]=\left\{\left|1_{13} 1_{24}\right\rangle,\left|8_{13} 8_{24}\right\rangle\right\}} \\
& {\left[\left(q_{1} \bar{q}_{4}\right)\left(q_{2} \bar{q}_{3}\right)\right]=\left\{\left|1_{14} 1_{23}\right\rangle,\left|8_{14} 8_{23}\right\rangle\right\} .}
\end{aligned}
$$

These two basis contain both singlet-singlet (physical) and octect-octect (hiddencolor) components. It is possible to prove that there is a minimum value for the octect-octect component probability of the wave function either in the $\left[\left(Q_{1} \bar{n}_{3}\right)\left(Q_{2} \bar{n}_{4}\right)\right]$ or the $\left[\left(Q_{1} \bar{n}_{4}\right)\left(Q_{2} \bar{n}_{3}\right)\right]$ couplings: $P_{88}^{13,24}, P_{88}^{14,23} \in[1 / 3,2 / 3]$. Does this imply an important hidden-color component in all $Q Q \bar{n} \bar{n}$ states? The answer is no ${ }^{14}$, because one can express any $Q Q \bar{n} \bar{n}$ state in terms of the singlet-singlet component of the $\left[\left(Q_{1} \bar{n}_{3}\right)\left(Q_{2} \bar{n}_{4}\right)\right]$ and $\left[\left(Q_{1} \bar{n}_{4}\right)\left(Q_{2} \bar{n}_{3}\right)\right]$ basis.

This discussion can be made more quantitative. Let us assume that $\{P, Q\}$ and $\{\hat{P}, \hat{Q}\}$ are the projectors associated to two orthonormal basis that are not orthogonal to each other, i.e., $P \hat{P}|\phi\rangle \neq 0$ and $P \hat{Q}|\phi\rangle \neq 0$ for an arbitrary state $|\phi\rangle$. This would be the case of the two orthonormal basis in Eq. (8). For any arbitrary state $|\Psi\rangle=P|\Psi\rangle+Q|\Psi\rangle$, the probability associated to $P$ or $\hat{P}$ will be given by $^{14}$,

$$
\mathcal{P}^{|\Psi\rangle}([u])=\frac{1}{2\left(1-\cos ^{2} \alpha\right)}[\langle\Psi|P \hat{Q}| \Psi\rangle+\langle\Psi|\hat{Q} P| \Psi\rangle]
$$




$$
\mathcal{P}^{|\Psi\rangle}\left(\left[u^{\prime}\right]\right)=\frac{1}{2\left(1-\cos ^{2} \alpha\right)}[\langle\Psi|\hat{P} Q| \Psi\rangle+\langle\Psi|Q \hat{P}| \Psi\rangle],
$$

where $P=|u\rangle\langle u|$ and $\hat{P}=\left|u^{\prime}\right\rangle\left\langle u^{\prime}\right|$ and $\cos \alpha=\left\langle u^{\prime} \mid u\right\rangle$. For a molecular state either $\mathcal{P}^{|\Psi\rangle}([u])$ or $\mathcal{P}^{|\Psi\rangle}\left(\left[u^{\prime}\right]\right)$ would be close to zero while for a compact state both will be different from zero.

We show in Table 1 the meson-meson probabilities for some selected four-quark states according to Ref. 14. Unbound states converge to two isolated mesons, the lowest threshold of the system, its root mean square radius (RMS) being very large. In contrast, bound states have a radius smaller than the threshold and they present probabilities different from zero for several physical states, the lowest two-meson threshold being contained in the physical four-quark system. Such states would be called compact in our notation. When the binding energy approaches the threshold, the probability of a single physical channel converges to one, what we defined as a molecular state. The $c c \bar{n} \bar{n} J^{P}(I)=1^{+}(0)$ is therefore a clear example of a real tetraquark, the r.m.s. of all Jacobi coordinates being small and with a significant probability of different vectors of the Hilbert space, pseudoscalar-vector $\left(M M^{*}\right)$ and vector-vector $\left(M^{*} M^{*}\right)$ components.

\section{Acknowledgments}

This work has been partially funded by the Spanish Ministerio de Educación y Ciencia and EU FEDER under Contracts No. FPA2007-65748 and FPA2010-21570, by Junta de Castilla y León under Contract No. GR12, and by the Spanish ConsoliderIngenio 2010 Program CPAN (CSD2007-00042),

\section{References}

1. M. Gell-Mann, Phys. Lett. 8, 214 (1964).

2. R. L. Jaffe, Phys. Rev. D 15, 267 (1977).

3. D. V. Bugg, Phys. Rep. 397, 257 (2004).

4. T. Fernández-Caramés, A. Valcarce and J. Vijande, Phys. Rev. Lett. 103, 222001 (2009).

5. T. F. Caramés, A. Valcarce and J. Vijande, Phys. Rev. D 82, 054032 (2010).

6. M. A. Moinester, Z. Phys. A 355, 349 (1996).

7. S. Zouzou, B. Silvestre-Brac, C. Gignoux and J.-M. Richard, Z. Phys. C 30, 457 (1986); L. Heller and J.A. Tjon, Phys. Rev. D 35, 969 (1987).

8. J. Vijande and A. Valcarce, Symmetry 1, 255 (2009).

9. R. K. Bhaduri, L. E. Cohler and Y. Nogami, Nuovo Cimento A65, 376 (1981).

10. J. Vijande, F. Fernández and A. Valcarce, J. Phys. G 31, 481 (2005).

11. J. Vijande, A. Valcarce and N. Barnea, Phys. Rev. D 79074010 (2009).

12. J. P. Ader, J.-M. Richard and P. Taxil, Phys. Rev. D 25, 2370 (1982).

13. J. Vijande, A. Valcarce and J.-M. Richard, Phys. Rev. D 76114013 (2007).

14. J. Vijande and A. Valcarce, Phys. Rev. C 80035204 (2009). 